\title{
THE ARAKAWA-KANEKO ZETA FUNCTION AND POLY-BERNOULLI POLYNOMIALS
}

\author{
YOSHINORI HAMAHATA
}

Ritsumeikan University, Japan

\begin{abstract}
The purpose of this paper is to introduce a generalization of the Arakawa-Kaneko zeta function and investigate their special values at negative integers. The special values are written as the sums of products of Bernoulli and poly-Bernoulli polynomials. We establish the basic properties for this zeta function and their special values.
\end{abstract}

\section{INTRODUCTION}

Let $\operatorname{Li}_{k}(x)(k \in \mathbb{Z})$ be the formal series defined by

$$
\operatorname{Li}_{k}(x)=\sum_{m=1}^{\infty} \frac{x^{m}}{m^{k}}
$$

The formal power series $\operatorname{Li}_{k}(x)$ is the $k$-th polylogarithm if $k \geq 1$, and a rational function if $k \leq 0$. When $k=1$, we see easily that

$$
\operatorname{Li}_{1}(x)=-\log (1-x) .
$$

The Arakawa-Kaneko zeta function $\xi_{k}(s, x)$, for $s \in \mathbb{C}, x>0, k \in \mathbb{Z}$, is defined by

$$
\xi_{k}(s, x)=\frac{1}{\Gamma(s)} \int_{0}^{\infty} \frac{\operatorname{Li}_{k}\left(1-e^{-t}\right)}{1-e^{-t}} e^{-x t} t^{s-1} d t .
$$

It is defined for $\operatorname{Re}(s)>0, x>0$ if $k \geq 1$, and for $\operatorname{Re}(s)>0, x>|k|+1$ if $k<$ 0 . The function $\xi_{k}(s, x)$ is a generalization of the Hurwitz zeta function $\zeta(s, x)$ in that $\xi_{1}(s, x)=s \zeta(s, x)$. Especially, $\xi_{k}(s):=\xi_{k}(s, 1)$, which was defined by Arakawa and Kaneko [1], is a generalization of the Riemann zeta function

2010 Mathematics Subject Classification. 11B68, $11 \mathrm{M} 32$.

Key words and phrases. Arakawa-Kaneko zeta function, Bernoulli numbers and polynomials, poly-Bernoulli numbers and polynomials. 
$\zeta(s)$ in that $\xi_{k}(s)=s \zeta(s+1)$. In this paper, we introduce a generalization of $\xi_{k}(s, x)$ and investigate their special values at negative integers.

To consider the special values of $\xi_{k}(s, x)$, we recall Bernoulli and polyBernoulli polynomials. Bernoulli polynomials $B_{n}(x)$ are defined by the generating function

$$
\frac{t e^{x t}}{e^{t}-1}=\sum_{n=0}^{\infty} B_{n}(x) \frac{t^{n}}{n !} .
$$

These polynomials are related to special values of the Hurwitz zeta function. There exist some relations among Bernoulli polynomials. For instance, the following identity is known:

(1.4) $\sum_{n=0}^{n}\left(\begin{array}{l}n \\ i\end{array}\right) B_{i}(x) B_{n-i}(y)=n(x+y-1) B_{n-1}(x+y)-(n-1) B_{n}(x+y)$

(see $[6,(3.2)]$ ). Dilcher [6] and Chen [4] gave a generalization of this identity for sums of products of Bernoulli polynomials given by

$$
\sum_{\substack{i_{1}+\cdots+i_{m}=n \\
i_{1}, \ldots, i_{m} \geq 0}}\left(\begin{array}{c}
n \\
i_{1}, \ldots, i_{m}
\end{array}\right) B_{i_{1}}\left(x_{1}\right) \cdots B_{i_{m-1}}\left(x_{m-1}\right) B_{i_{m}}\left(x_{m}\right)
$$

where

$$
\left(\begin{array}{c}
n \\
i_{1}, \ldots, i_{m}
\end{array}\right)=\frac{n !}{i_{1} ! \cdots i_{m} !}
$$

are multinomial coefficients.

We next recall poly-Bernoulli polynomials introduced in $[3,5]$. For every integer $k$, we define polynomials $B_{n}^{(k)}(x)$, which we call poly-Bernoulli polynomials, by

$$
\frac{\operatorname{Li}_{k}\left(1-e^{-t}\right)}{1-e^{-t}} e^{x t}=\sum_{n=0}^{\infty} B_{n}^{(k)}(x) \frac{t^{n}}{n !}
$$

We remark that $B_{n}^{(k)}(x)$ are defined in [5] by replacing $e^{x t}$ by $e^{-x t}$ in the left-hand side of (1.5). By definition, it is easy to see that for any $n \geq 0$

$$
B_{n}(x)=(-1)^{n} B_{n}^{(1)}(-x),
$$

or equivalently

$$
B_{n}^{(1)}(x)=B_{n}(x+1) .
$$

The numbers $B_{n}^{(k)}:=B_{n}^{(k)}(0)$ are called poly-Bernoulli numbers. These numbers are introduced by Kaneko [8], and then investigated in [1,2]. PolyBernoulli polynomials $B_{n}^{(k)}(x)$ were defined in [3] to generalize the properties of Bernoulli polynomials and poly-Bernoulli numbers. 
The purpose of this paper is to introduce a generalization of $\xi_{k}(s, x)$ and describe the special values at negative integers in terms of

$$
S_{m}^{(k)}(n ; x)=\sum_{\substack{i_{1}+\cdots+i_{m}=n \\
i_{1}, \ldots, i_{m} \geq 0}}\left(\begin{array}{c}
n \\
i_{1}, \ldots, i_{m}
\end{array}\right) B_{i_{1}}\left(x_{1}\right) \cdots B_{i_{m-1}}\left(x_{m-1}\right) B_{i_{m}}^{(k)}\left(x_{m}\right),
$$

where $x=x_{1}+\cdots+x_{n}$. Since $S_{m}^{(k)}(n ; x)$ is a generalization of $(1.5)$, it is of interest to investigate $S_{m}^{(k)}(n ; x)$. We show the outline of this paper. In Section 2, after some preparations of notations needed later, we present the basic properties for $S_{m}^{(k)}(n ; x)$. In Sections 3,4 and 5 , the proofs of these results are given. In Section 6 , we introduce a generalization of $\xi_{k}(s, x)$ and investigate the special values at negative integers in terms of $S_{m}^{(k)}(n ; x)$.

\section{Sums of products of Bernoulli And poly-Bernoulli POLYNOMIALS}

2.1. Preliminaries. Let $x_{1}, \ldots, x_{m}$ be variables and set $x=x_{1}+\cdots+x_{m}$. For $n \geq 0$, set

$$
S_{m}^{(k)}(n ; x)=\sum_{\substack{i_{1}+\cdots+i_{m}=n \\
i_{1}, \ldots, i_{m} \geq 0}}\left(\begin{array}{c}
n \\
i_{1}, \ldots, i_{m}
\end{array}\right) B_{i_{1}}\left(x_{1}\right) \cdots B_{i_{m-1}}\left(x_{m-1}\right) B_{i_{m}}^{(k)}\left(x_{m}\right),
$$

where $\left(\begin{array}{c}n \\ i_{1}, \ldots, i_{m}\end{array}\right)$ are multinomial coefficients defined by

$$
\left(\begin{array}{c}
n \\
i_{1}, \ldots, i_{m}
\end{array}\right)=\frac{n !}{i_{1} ! \cdots i_{m} !} .
$$

When $m=1, S_{m}^{(k)}(n)$ is nothing other than $B_{n}^{(k)}(x)$. By definition, we have

$$
\left(\frac{t}{e^{t}-1}\right)^{m-1} \frac{\operatorname{Li}_{k}\left(1-e^{-t}\right)}{1-e^{-t}} e^{x t}=\sum_{n=0}^{\infty} S_{m}^{(k)}(n ; x) \frac{t^{n}}{n !} .
$$

For $m \geq 1$, the Stirling numbers of the first kind $\left[\begin{array}{c}m \\ l\end{array}\right]$ are defined by

$$
x(x+1) \cdots(x+m-1)=\sum_{l=0}^{m}\left[\begin{array}{c}
m \\
l
\end{array}\right] x^{l}
$$

and $\left[\begin{array}{c}m \\ l\end{array}\right]=0$ for $l \geq m+1$ and $l \leq-1$. These numbers satisfy

$$
\begin{aligned}
{\left[\begin{array}{c}
m \\
0
\end{array}\right] } & =0, \quad\left[\begin{array}{l}
m \\
m
\end{array}\right]=1 \quad(m \geq 1) \\
{\left[\begin{array}{c}
m+1 \\
l
\end{array}\right] } & =\left[\begin{array}{c}
m \\
l-1
\end{array}\right]+m\left[\begin{array}{c}
m \\
l
\end{array}\right] \quad(m \geq 1, l \in \mathbb{Z}) .
\end{aligned}
$$


Let us introduce a differential operator by

$$
D(x)=\frac{d}{d t}-x .
$$

For the exponential generating function $\sum_{n=0}^{\infty} a_{n} t^{n} / n$ ! of a sequence $\left\{a_{n}\right\}$, it holds that

$$
D(x)\left(\sum_{n=0}^{\infty} a_{n} \frac{t^{n}}{n !}\right)=\sum_{n=0}^{\infty}\left(a_{n+1}-x a_{n}\right) \frac{t^{n}}{n !} .
$$

Fix $k \in \mathbb{Z}$. We denote by $P_{k}$ the set of $x^{l} B_{n}^{(k)}(x)(l, n \geq 0)$. Let $U$ and $V$ be maps of $P_{k}$ to itself with the conditions

$$
U\left(x^{l} B_{n}^{(k)}(x)\right)=x^{l} B_{n+1}^{(k)}(x), \quad V\left(x^{l} B_{n}^{(k)}(x)\right)=x^{l+1} B_{n}^{(k)}(x) .
$$

2.2. Results. We are now ready to state our results.

TheOREM 2.1. We have

$$
\begin{aligned}
& \sum_{l=0}^{m}(-1)^{m-l}\left[\begin{array}{c}
m+1 \\
l+1
\end{array}\right] S_{m+1}^{(k-l)}(n ; x) \\
& \quad= \begin{cases}\frac{n !}{(n-m) !} \sum_{l=1}^{m}\left[\begin{array}{c}
m+1 \\
l+1
\end{array}\right](U-V)^{l} B_{n-m}^{(k)}(x), & n \geq m \\
0, & 0 \leq n \leq m-1\end{cases}
\end{aligned}
$$

The proof is given in Section 3 .

EXAMPLE 2.2.

$$
\begin{aligned}
- & S_{2}^{(k)}(n ; x)+S_{2}^{(k-1)}(n ; x)=n B_{n}^{(k)}(x)-n x B_{n-1}^{(k)}(x) \quad(n \geq 1), \\
2 S_{3}^{(k)}(n ; x)-3 S_{3}^{(k-1)}(n ; x)+S_{3}^{(k-2)}(n ; x) & \\
\quad= & n(n-1)\left[B_{n}^{(k)}(x)+(3-2 x) B_{n-1}^{(k)}(x)+\left(x^{2}-3 x\right) B_{n-2}^{(k)}(x)\right] \quad(n \geq 2), \\
- & 6 S_{4}^{(k)}(n ; x)+11 S_{4}^{(k-1)}(n ; x)-6 S_{4}^{(k-2)}(n ; x)+S_{4}^{(k-3)}(n ; x) \\
& =n(n-1)(n-2)\left[B_{n}^{(k)}(x)+(6-3 x) B_{n-1}^{(k)}(x)\right. \\
& \left.\quad+\left(11-12 x+3 x^{2}\right) B_{n-2}^{(k)}(x)-\left(11 x-6 x^{2}+x^{3}\right) B_{n-3}^{(k)}(x)\right] \quad(n \geq 3) .
\end{aligned}
$$

TheOREM 2.3. For $k \geq 1$ and $n \geq 1$, we have

$$
\begin{aligned}
S_{2}^{(0)}(n ; x) & =B_{n}^{(1)}(x), \\
S_{2}^{(k)}(n ; x) & =B_{n}(x+1)-n \sum_{j=1}^{k} B_{n}^{(j)}(x)+n x \sum_{j=1}^{k} B_{n-1}^{(j)}(x), \\
S_{2}^{(-k)}(n ; x) & =B_{n}(x+1)+n \sum_{j=0}^{k-1} B_{n}^{(-j)}(x)+n x \sum_{j=0}^{k-1} B_{n-1}^{(-j)}(x) .
\end{aligned}
$$


The proof is given in Section 4.

ThEOREM 2.4. For $k \geq 1$ and $n \geq 2$, we have

$$
\begin{aligned}
S_{3}^{(0)}(n ; x)= & -(n-1) B_{n}^{(1)}(x)+n x B_{n-1}^{(1)}(x) \\
S_{3}^{(k)}(n ; x)= & \left(2-2^{-k}\right)\left[n x B_{n-1}(x)-(n-1) B_{n}(x)\right] \\
& -\left(1-2^{-k}\right)\left[n(x+1) B_{n-1}(x+1)\right. \\
& \left.-(n-1) B_{n}(x+1)\right] \\
& +n(n-1) \sum_{j=1}^{k}\left(1-2^{j-k-1}\right)\left[B_{n}^{(j)}(x)\right. \\
& \left.+(1-2 x) B_{n-1}^{(j)}(x)+\left(x^{2}-x\right) B_{n-2}^{(j)}(x)\right] \\
S_{3}^{(-k)}(n ; x)= & \left(2-2^{k}\right)\left[n x B_{n-1}(x)-(n-1) B_{n}(x)\right] \\
& -\left(1-2^{k}\right)\left[n(x+1) B_{n-1}(x+1)\right. \\
& \left.-(n-1) B_{n}(x+1)\right] \\
& +n(n-1) \sum_{j=1}^{k-2}\left(2^{k-j-1}-1\right)\left[B_{n}^{(-j)}(x)\right. \\
& \left.+(1-2 x) B_{n-1}^{(-j)}(x)+\left(x^{2}-x\right) B_{n-2}^{(-j)}(x)\right],
\end{aligned}
$$

where $S_{3}^{(-1)}(n ; x)=n(x+1) B_{n-1}(x+1)-(n-1) B_{n}(x+1)$.

The proof is given in Section 5 .

REMARK 2.5. It is difficult to find a formula for $S_{m}^{(k)}(n ; x)$ when $m \geq 4$.

2.3. Corollaries. We present some results derived from Theorems 2.1, 2.3 and 2.4 .

2.3.1. Sums of products of Bernoulli and poly-Bernoulli numbers. Let $k$ be an integer. For $m \geq 1$ and $n \geq 0$, set

$$
S_{m}^{(k)}(n)=\sum_{\substack{i_{1}+\cdots+i_{m}=n \\
i_{1}, \ldots, i_{m} \geq 0}}\left(\begin{array}{c}
n \\
i_{1}, \ldots, i_{m}
\end{array}\right) B_{i_{1}} \cdots B_{i_{m-1}} B_{i_{m}}^{(k)} .
$$

When $m=1, S_{m}^{(k)}(n)$ becomes $B_{n}^{(k)}$. By definition, we have

$$
\left(\frac{t}{e^{t}-1}\right)^{m-1} \frac{\operatorname{Li}_{k}\left(1-e^{-t}\right)}{1-e^{-t}}=\sum_{n=0}^{\infty} S_{m}^{(k)}(n) \frac{t^{n}}{n !} .
$$

Putting $x=0$ in Theorem 2.1, we have the following theorem. 
Theorem 2.6 (Kamano [7]). For $k \in \mathbb{Z}$ and $m \geq 1$, we have

$$
\begin{aligned}
& \sum_{l=0}^{m}(-1)^{m-l}\left[\begin{array}{c}
m+1 \\
l+1
\end{array}\right] S_{m+1}^{(k-l)}(n) \\
& =\left\{\begin{array}{ll}
n(n-1) \cdots(n-m+1) \sum_{l=1}^{m}\left[\begin{array}{c}
m \\
l
\end{array}\right] B_{n-m+l}^{(k)}, & n \geq m \\
0, & 0 \leq n \leq m-1
\end{array} .\right.
\end{aligned}
$$

Putting $x=0$ in Theorem 2.3, we obtain the following theorem.

Theorem 2.7 (Kamano [7]). For $k \geq 1$ and $n \geq 1$, we have

$$
\begin{aligned}
S_{2}^{(0)}(n) & =B_{n}^{(1)}, \\
S_{2}^{(k)}(n) & =B_{n}^{(1)}-n \sum_{j=1}^{k} B_{n}^{(j)}, \\
S_{2}^{(-k)}(n) & =B_{n}^{(1)}+n \sum_{j=0}^{k-1} B_{n}^{(-j)} .
\end{aligned}
$$

In Theorem 2.4, replacing $B_{n-1}(x+1)$ by $(-1)^{n-1} B_{n-1}(-x)$ and putting $x=0$, we obtain the following theorem.

TheOREM 2.8 (Kamano [7]). For $k \geq 1$ and $n \geq 2$, we have

$$
\begin{aligned}
S_{3}^{(0)}(n)= & -(n-1) B_{n}, \\
S_{3}^{(k)}(n)= & n\left(1-2^{-k}\right)(-1)^{n} B_{n-1}-(n-1) B_{n} \\
& +n(n-1) \sum_{j=1}^{k}\left(1-2^{j-k-1}\right)\left(B_{n}^{(j)}+B_{n-1}^{(j)}\right), \\
S_{3}^{(-k)}(n)= & n\left(2^{k}-1\right)(-1)^{n-1} B_{n-1}-(n-1) B_{n} \\
& +n(n-1) \sum_{j=0}^{k-2}\left(2^{k-j-1}-1\right)\left(B_{n}^{(-j)}+B_{n-1}^{(-j)}\right),
\end{aligned}
$$

where $S_{3}^{(-1)}(n)=n(-1)^{n-1} B_{n-1}-(n-1) B_{n}$.

REMARK 2.9. In the proofs of Theorems 2.6, 2.7 and 2.8, the operator $D(0)=d / d t$ was used. On the other hand, in the proofs of Theorems 2.1, 2.3 and $2.4, D(x)=d / d t-x$ will be used. 
2.3.2. Sums of products of Bernoulli polynomials. The identity (2.4) for $k=1$ turns into

$$
\begin{aligned}
\sum_{i=0}^{n}\left(\begin{array}{c}
n \\
i
\end{array}\right) B_{i}\left(x_{1}\right) & B_{n-i}\left(x_{2}+1\right) \\
& =n\left(x_{1}+x_{2}\right) B_{n-1}\left(x_{1}+x_{2}+1\right)-(n-1) B_{n}\left(x_{1}+x_{2}+1\right) .
\end{aligned}
$$

Putting $x=x_{1}$ and $y=x_{2}+1$, (1.4) is gained.

Similarly, using (2.7) for $k=1$ and $n \geq 2$, we obtain the following result:

$$
\begin{aligned}
& \sum_{\substack{i_{1}+i_{2}+i_{3}=n \\
i_{1}, i_{2}, i_{3} \geq 0}}\left(\begin{array}{c}
n \\
i_{1}, i_{2}, i_{3}
\end{array}\right) B_{i_{1}}\left(x_{1}\right) B_{i_{2}}\left(x_{2}\right) B_{i_{3}}\left(x_{3}\right) \\
& =\frac{n(n-1)}{2}(x-1)(x-2) B_{n-2}(x) \\
& \quad+\frac{n}{2}(3 n-3-2 n x+x) B_{n-1}(x)+\frac{3}{2} n(x-1) B_{n-1}(x-1) \\
& \quad+\frac{n^{2}-1}{2} B_{n}(x)-\frac{3}{2}(n-1) B_{n}(x-1) .
\end{aligned}
$$

\section{Proof of Theorem 2.1}

Let $G_{k}(t, x)$ be the generating function of poly-Bernoulli polynomials of index $k$ given by the left-hand side of (1.6). For example, we have

$$
G_{-1}(t, x)=e^{(x+2) t}, \quad G_{0}(t, x)=e^{(x+1) t}, \quad G_{1}(t, x)=\frac{t e^{(x+1) t}}{e^{t}-1} .
$$

The following lemma is a key result in the proofs of Theorems 2.1, 2.3 and 2.4 .

LEMma 3.1. For $k \in \mathbb{Z}$, we have

$$
D(x) G_{k}(t, x)=\frac{1}{e^{t}-1}\left(G_{k-1}(t, x)-G_{k}(t, x)\right) .
$$

Proof. For $F_{k}(t)=\operatorname{Li}_{k}\left(1-e^{-t}\right) /\left(1-e^{-t}\right)$, Kamano [7] proved

$$
\frac{d}{d t} F_{k}(t)=\frac{1}{e^{t}-1}\left(F_{k-1}(t)-F_{k}(t)\right) \text {. }
$$

From this, we deduce

$$
\frac{d}{d t} G_{k}(t, x)=\frac{1}{e^{t}-1}\left(G_{k-1}(t, x)-G_{k}(t, x)\right)+x G_{k}(t, x) .
$$

Let us generalize the lemma just proved. 
TheOrem 3.2. For $k \in \mathbb{Z}$ and $m \geq 1$, it holds that

$$
\begin{gathered}
\left(\left[\begin{array}{l}
m \\
m
\end{array}\right] D(x)^{m}+\left[\begin{array}{c}
m \\
m-1
\end{array}\right] D(x)^{m-1}+\cdots+\left[\begin{array}{c}
m \\
1
\end{array}\right] D(x)\right) G_{k}(t, x) \\
=\frac{1}{\left(e^{t}-1\right)^{m}} \sum_{l=0}^{m}(-1)^{m-l}\left[\begin{array}{c}
m+1 \\
l+1
\end{array}\right] G_{k-l}(t, x) .
\end{gathered}
$$

Proof. We prove the theorem by induction on $m$. The case $m=1$ follows from the lemma stated above. Assume that (3.2) holds for case $m$. By $(2.2)$, the left-hand side of $(3.2)$ for case $m+1$ is

$$
\begin{aligned}
\left(\left[\begin{array}{l}
m \\
m
\end{array}\right] D(x)^{m+1}+\left[\begin{array}{c}
m \\
m-1
\end{array}\right]\right. & \left.D(x)^{m}+\cdots+\left[\begin{array}{c}
m \\
1
\end{array}\right] D(x)^{2}\right) G_{k}(t, x) \\
& +m\left(\left[\begin{array}{l}
m \\
m
\end{array}\right] D(x)^{m}+\cdots+\left[\begin{array}{c}
m \\
1
\end{array}\right] D(x)\right) G_{k}(t, x) .
\end{aligned}
$$

Thanks to the assumption for case $m$, this becomes

$$
\begin{aligned}
D(x)\left(\frac{1}{\left(e^{t}-1\right)^{m}} \sum_{l=0}^{m}(-1)^{m-l}\right. & {\left.\left[\begin{array}{c}
m+1 \\
l+1
\end{array}\right] G_{k-l}(t, x)\right) } \\
& +\frac{m}{\left(e^{t}-1\right)^{m}} \sum_{l=0}^{m}(-1)^{m-l}\left[\begin{array}{c}
m+1 \\
l+1
\end{array}\right] G_{k-l}(t, x) .
\end{aligned}
$$

Applying Lemma 3.1 to the first term gives

$$
\begin{aligned}
& \frac{-m e^{t}}{\left(e^{t}-1\right)^{m+1}} \sum_{l=0}^{m}(-1)^{m-l}\left[\begin{array}{c}
m+1 \\
l+1
\end{array}\right] G_{k-l}(t, x) \\
& +\frac{1}{\left(e^{t}-1\right)^{m+1}} \sum_{l=0}^{m}(-1)^{m-l}\left[\begin{array}{c}
m+1 \\
l+1
\end{array}\right]\left(G_{k-l-1}(t, x)-G_{k-l}(t, x)\right) \\
& +\frac{m}{\left(e^{t}-1\right)^{m}} \sum_{l=0}^{m}(-1)^{m-l}\left[\begin{array}{c}
m+1 \\
l+1
\end{array}\right] G_{k-l}(t, x) \\
& =\frac{-m-1}{\left(e^{t}-1\right)^{m+1}} \sum_{l=0}^{m}(-1)^{m-l}\left[\begin{array}{c}
m+1 \\
l+1
\end{array}\right] G_{k-l}(t, x) \\
& +\frac{1}{\left(e^{t}-1\right)^{m+1}} \sum_{l=0}^{m}(-1)^{m-l}\left[\begin{array}{c}
m+1 \\
l+1
\end{array}\right] G_{k-l-1}(t, x) \\
& =\frac{1}{\left(e^{t}-1\right)^{m+1}} \sum_{l=0}^{m}(-1)^{m+1-l}(m+1)\left[\begin{array}{c}
m+1 \\
l+1
\end{array}\right] G_{k-l}(t, x) \\
& +\frac{1}{\left(e^{t}-1\right)^{m+1}} \sum_{l=1}^{m+1}(-1)^{m+1-l}\left[\begin{array}{c}
m+1 \\
l
\end{array}\right] G_{k-l}(t, x) .
\end{aligned}
$$


Using $\left[\begin{array}{l}m+1 \\ m+2\end{array}\right]=\left[\begin{array}{c}m+1 \\ 0\end{array}\right]=0$, the right-hand side turns into

$$
\frac{1}{\left(e^{t}-1\right)^{m+1}} \sum_{l=0}^{m+1}(-1)^{m+1-l}\left((m+1)\left[\begin{array}{c}
m+1 \\
l+1
\end{array}\right]+\left[\begin{array}{c}
m+1 \\
l
\end{array}\right]\right) G_{k-l}(t, x),
$$

which yields the claim for case $m+1$.

Let us return to the proof of Theorem 2.1. We see that

$$
\begin{aligned}
D(x) G_{k}(t, x) & =\sum_{n=1}^{\infty} B_{n}^{(k)}(x) \frac{t^{n-1}}{(n-1) !}-x \sum_{n=0}^{\infty} B_{n}^{(k)}(x) \frac{t^{n}}{n !} \\
& =\sum_{n=0}^{\infty}(U-V) B_{n}^{(k)}(x) \frac{t^{n}}{n !} .
\end{aligned}
$$

Since $D(x)$ and $U-V$ are commutative, for $l \geq 0$ we obtain

$$
D(x)^{l} G_{k}(t, x)=\sum_{n=0}^{\infty}(U-V)^{l} B_{n}^{(k)}(x) \frac{t^{n}}{n !} .
$$

For $m \geq 0$, we have

$$
t^{m} D(x)^{l} G_{k}(t, x)=\sum_{n=m}^{\infty} \frac{n !}{(n-m) !}(U-V)^{l} B_{n-m}^{(k)}(x) \frac{t^{n}}{n !} .
$$

Therefore the theorem follows from Theorem 3.2.

\section{Proof of Theorem 2.3}

Proof of (2.3): This case follows from $G_{0}(t, x)=e^{(x+1) t}$.

Proof of (2.4): By Lemma 3.1,

$$
\frac{d}{d t} G_{j}(t, x)=\frac{1}{e^{t}-1}\left(G_{j-1}(t, x)-G_{j}(t, x)\right)+x G_{j}(t, x) .
$$

Summing over $j$ from 1 to $k$, we have

$$
\sum_{j=1}^{k} \frac{d}{d t} G_{j}(t, x)=\frac{1}{e^{t}-1}\left(G_{0}(t, x)-G_{k}(t, x)\right)+x \sum_{j=1}^{k} G_{j}(t, x),
$$

or equivalently

$$
\frac{1}{e^{t}-1} G_{k}(t, x)=\frac{e^{(x+1) t}}{e^{t}-1}-\sum_{j=1}^{k} \frac{d}{d t} G_{j}(t, x)+x \sum_{j=1}^{k} G_{j}(t, x) .
$$


Multiplying by $t$, we have

$$
\begin{aligned}
\frac{t}{e^{t}-1} G_{k}(t, x)= & \sum_{n=0}^{\infty} B_{n}(x+1) \frac{t^{n}}{n !}-\sum_{n=1}^{\infty} n \sum_{j=1}^{k} B_{n}^{(k)}(x) \frac{t^{n}}{n !} \\
& +x \sum_{n=1}^{\infty} n \sum_{j=1}^{k} B_{n-1}^{(j)}(x) \frac{t^{n}}{n !},
\end{aligned}
$$

which yields the result.

Proof of (2.5): By Lemma 3.1,

$$
\frac{d}{d t} G_{-j}(t, x)=\frac{1}{e^{t}-1}\left(G_{-j-1}(t, x)-G_{-j}(t, x)\right)+x G_{-j}(t, x) .
$$

Summing over $j$ from 0 to $k-1$, we get

$$
\sum_{j=0}^{k-1} \frac{d}{d t} G_{-j}(t, x)=\frac{1}{e^{t}-1}\left(G_{-k}(t, x)-G_{0}(t, x)\right)+x \sum_{j=0}^{k-1} G_{-j}(t, x),
$$

or equivalently

$$
\frac{1}{e^{t}-1} G_{-k}(t, x)=\frac{e^{(x+1) t}}{e^{t}-1}+\sum_{j=0}^{k-1} \frac{d}{d t} G_{-j}(t, x)-x \sum_{j=0}^{k-1} G_{-j}(t, x) .
$$

Multiplying both sides of this identity by $t$, we have the result.

\section{Proof of Theorem 2.4}

Proof of (2.6): Using $G_{0}(t, x)=e^{(x+1) t}$ and $G_{1}(t, x)=t e^{(x+1) t} /\left(e^{t}-1\right)$,

$$
\frac{t^{2}}{\left(e^{t}-1\right)^{2}} G_{0}(t, x)=\frac{t}{e^{t}-1} G_{1}(t, x),
$$

which implies

$$
S_{3}^{(0)}(n, x)=S_{2}^{(1)}(n ; x)=B_{n}(x+1)-n B_{n}^{(1)}(x)+n x B_{n-1}^{(1)}(x) .
$$

By (1.8), we obtain (2.6).

Proof of (2.7): By Theorem 3.2 for $m=2$, we have

$$
\begin{aligned}
\left(D(x)^{2}\right. & +D(x)) G_{j}(t, x) \\
& =\frac{1}{\left(e^{t}-1\right)^{2}}\left[\left(2 G_{j}(t, x)-G_{j-1}(t, x)\right)-\left(2 G_{j-1}(t, x)-G_{j-2}(t, x)\right)\right] .
\end{aligned}
$$

Summing over $j$ from 1 to $l$,

$$
\frac{2 G_{l}(t, x)}{\left(e^{t}-1\right)^{2}}-\frac{G_{l-1}(t, x)}{\left(e^{t}-1\right)^{2}}=\frac{2 G_{0}(t, x)-G_{-1}(t, x)}{\left(e^{t}-1\right)^{2}}+\sum_{j=1}^{l}\left(D(x)^{2}+D(x)\right) G_{j}(t, x) .
$$


Multiplying both sides by $2^{l-1}$ and summing over $l$ from 1 to $k$, we have

$$
\begin{aligned}
\frac{2^{k} G_{k}(t, x)}{\left(e^{t}-1\right)^{2}}-\frac{G_{0}(t, x)}{\left(e^{t}-1\right)^{2}}= & \left(\sum_{l=1}^{k} 2^{l-1}\right) \frac{2 G_{0}(t, x)-G_{-1}(t, x)}{\left(e^{t}-1\right)^{2}} \\
& +\sum_{l=1}^{k} 2^{l-1} \sum_{j=1}^{l}\left(D(x)^{2}+D(x)\right) G_{j}(t, x) \\
= & \frac{\left(2^{k+1}-2\right) G_{0}(t, x)}{\left(e^{t}-1\right)^{2}}-\frac{\left(2^{k}-1\right) G_{-1}(t, x)}{\left(e^{t}-1\right)^{2}} \\
& +\sum_{j=1}^{k} \sum_{l=j}^{k} 2^{l-1}\left(D(x)^{2}+D(x)\right) G_{j}(t, x) .
\end{aligned}
$$

Using $G_{0}(t, x)=e^{(x+1) t}$ and $G_{-1}(t, x)=e^{(x+2) t}$, it holds that

$$
\begin{aligned}
\frac{G_{k}(t, x)}{\left(e^{t}-1\right)^{2}}= & \left(2-2^{-k}\right) \frac{e^{(x+1) t}}{\left(e^{t}-1\right)^{2}}-\left(1-2^{-k}\right) \frac{e^{(x+2) t}}{\left(e^{t}-1\right)^{2}} \\
& +\sum_{j=1}^{k}\left(1-2^{j-k-1}\right)\left(D(x)^{2}+D(x)\right) G_{j}(t, x) .
\end{aligned}
$$

We multiply both sides by $t^{2}$, and calculate each term of the right-hand side:

$$
\begin{gathered}
\text { (5.1) } \frac{t^{2} e^{(x+1) t}}{\left(e^{t}-1\right)^{2}}=1+\sum_{n=1}^{\infty}\left(n x B_{n-1}(x)-(n-1) B_{n}(x)\right) \frac{t^{n}}{n !}, \\
\text { (5.2) } \quad \frac{t^{2} e^{(x+2) t}}{\left(e^{t}-1\right)^{2}}=1+\sum_{n=1}^{\infty}\left(n(x+1) B_{n-1}(x+1)-(n-1) B_{n}(x+1)\right) \frac{t^{n}}{n !}, \\
\text { (5.3) } \quad t^{2} D(x)^{2} G_{j}(t, x) \\
=\sum_{n=2}^{\infty} n(n-1)\left(B_{n}^{(j)}(x)-2 x B_{n-1}^{(j)}(x)+x^{2} B_{n-2}^{(j)}(x)\right) \frac{t^{n}}{n !}, \\
\text { (5.4) } \quad t^{2} D(x) G_{j}(t, x)=\sum_{n=2}^{\infty} n(n-1)\left(B_{n-1}^{(j)}(x)-x B_{n-2}^{(j)}(x)\right) \frac{t^{n}}{n !} .
\end{gathered}
$$

Here (5.1) follows from

$$
\frac{t^{2} e^{(x+1) t}}{\left(e^{t}-1\right)^{2}}=\frac{t e^{x t}}{e^{t}-1}+x t \cdot \frac{t e^{x t}}{e^{t}-1}-t \frac{d}{d t}\left(\frac{t e^{x t}}{e^{t}-1}\right) .
$$

From these, when $n \geq 2$, we obtain (2.7). 
Proof of (2.8): Using Theorem 3.2 for $m=2$, we have

$$
\begin{aligned}
& \left(D(x)^{2}+D(x)\right) G_{-j}(t, x) \\
& =\frac{1}{\left(e^{t}-1\right)^{2}}\left[\left(2 G_{-j}(t, x)-G_{-j-1}(t, x)\right)-\left(2 G_{-j-1}(t, x)-G_{-j-2}(t, x)\right)\right] .
\end{aligned}
$$

Summing over $j$ from 0 to $l$,

$$
\begin{aligned}
\frac{2 G_{-l-1}(t, x)}{\left(e^{t}-1\right)^{2}}-\frac{G_{-l-2}(t, x)}{\left(e^{t}-1\right)^{2}}= & \frac{2 G_{0}(t, x)-G_{-1}(t, x)}{\left(e^{t}-1\right)^{2}} \\
& -\sum_{j=0}^{l}\left(D(x)^{2}+D(x)\right) G_{-j}(t, x) .
\end{aligned}
$$

Multiplying both sides by $2^{-l}$ and summing over $l$ from 0 to $k-2$, we have

$$
\begin{aligned}
& \frac{2 G_{-1}(t, x)}{\left(e^{t}-1\right)^{2}}-\frac{2^{-k+2} G_{-k}(t, x)}{\left(e^{t}-1\right)^{2}} \\
& =\left(\sum_{l=0}^{k-2} 2^{-l}\right) \frac{2 G_{0}(t, x)-G_{-1}(t, x)}{\left(e^{t}-1\right)^{2}}-\sum_{l=0}^{k-2} 2^{-l} \sum_{j=0}^{l}\left(D(x)^{2}+D(x)\right) G_{-j}(t, x) \\
& =\frac{2^{2}\left(1-2^{-k+1}\right) G_{0}(t, x)}{\left(e^{t}-1\right)^{2}}-\frac{2\left(1-2^{-k+1}\right) G_{-1}(t, x)}{\left(e^{t}-1\right)^{2}} \\
& \quad-\sum_{j=0}^{k-2} \sum_{l=j}^{k-2} 2^{-l}\left(D(x)^{2}+D(x)\right) G_{-j}(t, x) .
\end{aligned}
$$

Using $G_{0}(t, x)=e^{(x+1) t}$ and $G_{-1}(t, x)=e^{(x+2) t}$, we obtain

$$
\begin{aligned}
\frac{G_{-k}(t, x)}{\left(e^{t}-1\right)^{2}}= & \left(2-2^{k}\right) \frac{e^{(x+1) t}}{\left(e^{t}-1\right)^{2}}-\left(1-2^{k}\right) \frac{e^{(x+2) t}}{\left(e^{t}-1\right)^{2}} \\
& +\sum_{j=0}^{k-2}\left(2^{k-j-1}-1\right)\left(D(x)^{2}+D(x)\right) G_{-j}(t, x) .
\end{aligned}
$$

We multiply both sides by $t^{2}$, and calculate each term of the right-hand side: above all, we see easily that

$$
\begin{aligned}
& t^{2} D(x)^{2} G_{-j}(t, x) \\
& \quad=\sum_{n=2}^{\infty} n(n-1)\left(B_{n}^{(-j)}(x)-2 x B_{n-1}^{(-j)}(x)+x^{2} B_{n-2}^{(-j)}(x)\right) \frac{t^{n}}{n !}, \\
& t^{2} D(x) G_{-j}(t, x)=\sum_{n=2}^{\infty} n(n-1)\left(B_{n-1}^{(-j)}(x)-x B_{n-2}^{(-j)}(x)\right) \frac{t^{n}}{n !} .
\end{aligned}
$$

Combining (5.1), (5.2), (5.5), (5.6) with right-hand side of $G_{-k}(t, x) /\left(e^{t}-1\right)^{2}$, the identity (2.8) can be established for $n \geq 2$. 


\section{A generalization of the Arakawa-Kaneko zeta function}

Let $k$ be an integer and $m$ be a positive integer. We introduce zeta functions by means of the Laplace-Mellin integral.

Definition 6.1. For $k \in \mathbb{Z}$ and $m \geq 1$, define

$$
\begin{aligned}
\xi_{k, m}(s, x) & =\frac{1}{\Gamma(s)} \int_{0}^{\infty}\left(\frac{t}{e^{t}-1}\right)^{m-1} \frac{L i_{k}\left(1-e^{-t}\right)}{1-e^{-t}} e^{-x t} t^{s-1} d t \\
\xi_{k, m}(s) & =\xi_{k, m}(s, 1)=\frac{1}{\Gamma(s)} \int_{0}^{\infty}\left(\frac{t}{e^{t}-1}\right)^{m-1} \frac{L i_{k}\left(1-e^{-t}\right)}{e^{t}-1} t^{s-1} d t .
\end{aligned}
$$

The zeta function $\xi_{k, m}(s, x)$ is defined for $\operatorname{Re}(s)>0$ and $x>0$ if $k \geq 1$, and for $\operatorname{Re}(s)>0$ and $x>|k|+1$ if $k \leq 0$. Hence $\xi_{k, m}(s)$ is defined for $\operatorname{Re}(s)>0$ and $k \geq 1$. It should be noted that $\xi_{k, 1}(s, x)$ is just the zeta function $\xi_{k}(s, x)$, and $\xi_{k, 1}(s)$ is the zeta function $\xi_{k}(s)$ defined in Section 1.

TheOREM 6.2. When $k \geq 1$ (resp. $k \leq 0$ ), suppose $x>0$ (resp. $x>$ $|k|+1)$. Then the function $s \mapsto \xi_{k, m}(s, x)$ can be analytically continued to the whole complex s-plane as an entire function and its values at negative integers are given by

$$
\xi_{k, m}(-n, x)=(-1)^{n} S_{m}^{(k)}(n ;-x) \quad(n=1,2,3, \ldots) .
$$

Proof. We express $\xi_{k, m}(s, x)$ as the sum of two integrals:

$$
\begin{aligned}
\xi_{k, m}(s, x)= & \frac{1}{\Gamma(s)} \int_{0}^{1}\left(\frac{t}{e^{t}-1}\right)^{m-1} \frac{\operatorname{Li}_{k}\left(1-e^{-t}\right)}{1-e^{-t}} e^{-x t} t^{s-1} d t \\
& +\frac{1}{\Gamma(s)} \int_{1}^{\infty}\left(\frac{t}{e^{t}-1}\right)^{m-1} \frac{\operatorname{Li}_{k}\left(1-e^{-t}\right)}{1-e^{-t}} e^{-x t} t^{s-1} d t .
\end{aligned}
$$

For any $s \in \mathbb{C}$, the second integral converges absolutely and the second term on the right-hand side becomes zero thanks to $\Gamma(s)^{-1}$. If $\operatorname{Re}(s)>0$, then the first term on the right-hand side is written as

$$
\frac{1}{\Gamma(s)} \sum_{i=0}^{\infty} \frac{S_{m}^{(k)}(i ;-x)}{i !} \cdot \frac{1}{i+s}
$$

From this, for a non-negative integer $n$, we get

$$
\xi_{k, m}(-n, x)=\left(\lim _{s \rightarrow-n} \frac{1}{\Gamma(s)(n+s)}\right) \frac{S_{m}^{(k)}(n ;-x)}{n !}=(-1)^{n} S_{m}^{(k)}(n ;-x) .
$$

Letting $x=1$ in Theorem 6.2, we get an extension of a result by ArakawaKaneko (see [1, Theorem 6 (i)]). 
TheOREM 6.3. Assume $k \geq 1$ and $x>0$. Then the function $s \mapsto$ $\xi_{k, m}(s, x)$ can be analytically continued to the whole complex s-plane as an entire function and its values at negative integers are given by

$$
\xi_{k, m}(-n)=\sum_{r=0}^{n}(-1)^{r}\left(\begin{array}{l}
n \\
r
\end{array}\right) S_{m}^{(k)}(r) \quad(n=1,2,3, \ldots) .
$$

Proof. The part of analytic continuation of the zeta function follows from the last theorem. Noting the generating function of $S_{m}^{(k)}(n ;-1)$ is

$$
\left(\sum_{n=0}^{\infty} S_{m}^{(k)}(n) \frac{t^{n}}{n !}\right) e^{-t}=\sum_{n=0}^{\infty}\left(\sum_{r=0}^{n}(-1)^{n-r}\left(\begin{array}{c}
n \\
r
\end{array}\right) S_{m}^{(k)}(r)\right) \frac{t^{n}}{n !},
$$

we have the result about special values from the last theorem.

We conclude this section by giving a few identities for $\xi_{k, m}(s, x)$.

THEOREM 6.4 (Difference identity). Let $m \geq 2$. With the hypothesis of Theorem 6.2, we have

$$
\xi_{k, m}(s, x+1)-\xi_{k, m}(s, x)=-s \xi_{k, m-1}(s+1, x) .
$$

Proof. The left-hand side becomes

$$
-\frac{1}{\Gamma(s)} \int_{0}^{\infty}\left(\frac{t}{e^{t}-1}\right)^{m-1} \frac{\operatorname{Li}_{k}\left(1-e^{-t}\right)}{1-e^{-t}} e^{-x t} t^{s+1} d t,
$$

which is the right-hand side.

Putting $s=-n$ in (6.1), we obtain the following.

Corollary 6.5. Let $m \geq 2$. With the hypothesis of Theorem 6.2, we have

$$
S_{m}^{(k)}(n ;-x-1)-S_{m}^{(k)}(n ;-x)=-n S_{m-1}^{(k)}(n-1 ;-x) \quad(n=1,2,3, \ldots) .
$$

TheOrem 6.6 (Raabe's identity). Let $m \geq 2$. With the hypothesis of Theorem 6.2, we have

$$
\begin{aligned}
\int_{0}^{1} \xi_{k, m}(s, x+w) d w & =\xi_{k, m-1}(s, x+1), \\
\int_{0}^{1} S_{m}^{(k)}(n ;-x-w) d w & =S_{m-1}^{(k)}(n ;-x-1) \quad(n=1,2,3, \ldots) .
\end{aligned}
$$

Proof. As for (6.2), the left-hand side is

$$
\frac{1}{\Gamma(s)} \int_{0}^{\infty}\left(\frac{t}{e^{t}-1}\right)^{m-1} \frac{\operatorname{Li}_{k}\left(1-e^{-t}\right)}{1-e^{-t}}\left(\int_{0}^{1} e^{-(x+w) t} d w\right) t^{s-1} d t,
$$

which is equal to $\xi_{k, m}(s, x+1)$.

As for (6.3), it is sufficient to combine Theorem 6.2 and (6.2) for $s=-n$. 


\section{REFERENCES}

[1] T. Arakawa and M. Kaneko, Multiple zeta values, poly-Bernoulli numbers, and related zeta functions, Nagoya Math. J. 153 (1999), 189-209.

[2] T. Arakawa and M. Kaneko, On poly-Bernoulli numbers, Comment. Math. Univ. St. Paul. 48 (1999), 159-167.

[3] A. Bayad and Y. Hamahata, Polylogarithms and poly-Bernoulli polynomials, Kyushu J. Math. 65 (2011), 15-24.

[4] K.-W. Chen, Sums of products of generalized Bernoulli polynomials, Pacific J. Math. 208 (2003), 39-52.

[5] M.-A. Coppo and B. Candelpergher, The Arakawa-Kaneko zeta function, Ramanujan J. 22 (2010), 153-162.

[6] K. Dilcher, Sums of products of Bernoulli numbers, J. Number Theory 60 (1996), $23-41$.

[7] K. Kamano, Sums of products of Bernoulli numbers, including poly-Bernoulli numbers, J. Integer Seq. 13 (2010), Article 10.5.2.

[8] M. Kaneko, Poly-Bernoulli numbers, J. Théor. Nombres Bordeaux 9 (1997), 221-228.

Y. Hamahata

Institute for Teaching and Learning

Ritsumeikan University

1-1-1 Nojihigashi, Kusatsu, Shiga 525-8577

Japan

E-mail: hamahata@fc.ritsumei.ac.jp

Received: 31.1.2013. 